Diabetologe $2021 \cdot 17: 275-282$

https://doi.org/10.1007/s11428-020-00700-0

Online publiziert:27. November 2020

(c) Springer Medizin Verlag $\mathrm{GmbH}$, ein Teil von Springer Nature 2020
Lutz Heinemann' - Diana Drossel $\left.\right|^{2}$. Matthias Kaltheuner ${ }^{3}$

'Science Consulting in Diabetes GmbH, Neuss, Deutschland

${ }^{2}$ Diabetesbeauftragte des Deutschen Blinden- und Sehbehindertenverband (DBSV), Berlin, Deutschland

${ }^{3}$ Diabetologikum Leverkusen, Leverkusen, Deutschland

\section{DiaDigital, Apps und digitale Gesundheitsanwendungen}

\section{Resümee nach 5 Jahren und wie weiter?}

Nachdem es in Deutschland über eine Reihe von Jahren mehr Aufforderungen zur verstärkten Nutzung von digitalen Optionen bei der Betreuung von Patienten mit Diabetes $(\mathrm{PmD})$ gegeben hat, kam der entscheidende Anschub Anfang 2020 in Form eines kleinen Stücks mRNA („messenger“ Ribonukleinsäure). Es ist beachtlich $\mathrm{zu}$ sehen, wie relativ schnell und flexibel alle Beteiligten auf die Corona-Krise reagiert haben. Als der Bedarf da war, kam es innerhalb von Tagen zur Etablierung von telemedizinischen Betreuungsoptionen, die Schulung erfolgte virtuell etc. Auch die Kostenerstattung für die therapeutische Betreuung von $\mathrm{PmD}$ unter solchen Bedingungen wurde vergleichsweise rasch und unbürokratisch geändert. Ob all diese Veränderungen Bestand haben, bleibt abzuwarten, es war ja auch nicht alles - aber doch sehr vieles - positiv, was in dieser Stresssituation geschehen ist.

Klar ist aber geworden, PmD brauchen gerade auch in einer solchen beängstigenden und verunsichernden Situation eine belastbare Kommunikationsoption mit ihrem Diabetologen/dem Diabetesteam. Bei einem persönlichen Gespräch mit dem behandelnden Arzt in dessen Praxis gibt es deutlich mehr Interaktion, aber die Besprechung von CGM(kontinuierliches Glukosemonitoring)-Profilen und den sich daraus ableitenden Therapieanpassungen geht eben auch per Videokonferenz gut. Die Datenspeicherung in der Cloud, die verfügbaren Software-Optionen, Smartphones etc. bieten eine IT-Umgebung, von der vor einigen Jahren nur zu träumen war!

Im Folgenden soll in einem - durchaus kritischen - Ansatz die Entwicklung der letzten Jahre reflektiert werden, und Optionen/Wege sollen nach vorne skizziert werden. Hervorzuheben sind die Veränderungen in der Gesundheitspolitik in den letzten Jahren. Nachdem dort außer Ankündigungen über Jahre (Jahrzehnte?) eher wenig Konkretes in Hinsicht auf vermehrten Einsatz von digitalen Optionen im Bereich der Betreuung von kranken Menschen erfolgt ist, hat es in den letzten Jahren deutliche Entwicklungen gegeben, die auch andere Bereiche erfasst hat, so z. B. die Krankenversicherungen. Das Beharrungsvermögen von etablierten Strukturen ist erheblich, der Veränderungsdruck, den die Corona-Krise ausgelöst hat, aber auch. Wenn die Gesundheitspolitik in Abstimmung mit den zuständigen Fachgesellschaften und berufspolitischen Verbänden die Rahmenbedingungen für die Betreuung von PmD an die aktuellen Anforderungen anpasst - mit einem geeigneten „Zukunftsfenster“ -, dann sollte eine gute und sichere Betreuung von PmD weiterhin gewährleistet sein.

\section{Einsatz von Apps durch Patienten mit Diabetes}

Ein wichtiger Grund für die heute de facto flächendeckende Verbreitung von Smartphones ist, dass „kleine“ Programme auf diesen laufen können, die gezielt Aufgaben wahrnehmen können, die sog. Apps. Es gibt mittlerweile Hun- derte, wenn nicht Tausende von Apps nur für den Diabetesbereich in den AppStores der großen Anbieter von Betriebssystemen [1]. Dabei verdienen wohl nur wenige der Entwickler Geld mit diesen Apps, auch weil nur sehr wenige Apps konkret und längerfristig nach dem Runterladen genutzt werden. Es fehlen (öffentlich verfügbare) Analysen dazu, welche Apps in welchem Ausmaß genutzt werden. Damit eine hohe Nutzung erreicht wird, muss der Patient einen konkreten Vorteil davon im Alltag haben, die App muss ein Problem lösen. Der Support, den die App bietet, muss für den Nutzer in seinen Umgang mit der Erkrankung integrierbar sein. Die „emotionale“ Ansprache des Patienten durch die App ist anscheinend entscheidend für die Nutzungsdauer/-intensität, seine „subjektive Realität“ ist die Realität! In diesem Sinne „spürt“ der Patient die Sinnhaftigkeit einer App für sich recht schnell. Wichtiges Kriterium für den Erfolg einer App ist deren „einfache“ Nutzbarkeit. Dabei ist es ausgesprochen anspruchsvoll, die Bedienung einer App so $\mathrm{zu}$ gestalten, dass der Nutzer dies so wahrnimmt. In der Kommunikation zwischen Patient und Diabetologe wird das Thema Apps wohl auch nicht systematisch angesprochen; vielleicht auch deshalb, weil der damit verbundene Zeitaufwand nicht honoriert wird. Hierbei wird die Haltung des behandelnden Arztes zu Apps vermutlich auch einen Einfluss haben. Wenn es im Gespräch mit dem Diabetologen überhaupt um diesen Themenkomplex geht, dann ist eine von Patienten viel gestellte Frage 
die zu dem Thema Datensicherheit bei Medizinprodukten.

Grob unterteilt sollen die Apps PmD bei ihrem Diabetesmanagement helfen, indem sie diese bei der Dokumentation und Analyse der bei Diabetes massenhaft anfallenden Daten unterstützen, aber auch bei der Überwachung und Kontrolle der therapeutischen Schritte, der Dokumentation der Ernährung und des Bewegungsverhaltens [2]. Es gibt Themenbereiche bei der Betreuung von $\mathrm{PmD}$, für die immer noch geeignete Apps fehlen, so bei der von Frauen mit einem Gestationsdiabetes! Ein weiteres wichtiges Einsatzgebiet von Apps ist die Geräteanbindung, d.h. die Steuerung und auch schon die Regelung der verschiedenen technischen Systeme, die bei der Diabetestherapie eingesetzt werden, d.h. Systemen für die Blutglukoseselbstmessung (BGSM), zum kontinuierlichen Glukosemonitoring (CGM) und zur Insulinapplikation (sei es mit Smartpens oder Insulinpumpen). Bei der Kopplung von z. B. einem CGM-System und einer Insulinpumpe kann ein geschlossener Regelkreis aufgebaut werden, d.h. durch eine Rechenvorschrift (= Algorithmus) werden die kontinuierlich zur Verfügung stehenden Glukosewerte so in eine unmittelbare Anpassung der Insulininfusion ins subkutane Fettgewebe „übersetzt“, dass eigentlich die Glukosekonzentration immer im Zielbereich (= Time-in-Range [TiR]) sein sollte. Die hiermit verbundene Steuerung der Geräte erfolgte bisher lokal, d.h. nur über das Smartphone. Es gibt aber zunehmend Ansätze dazu, die bei Systemen zur automatisierten Insulindosierung (AID) anfallenden Daten direkt in die Cloud zu senden bzw. bei Bedarf von extern in die Funktionsweise der AID-Systeme eingreifen zu können.

\section{》) Wichtiges Kriterium für den Erfolg einer App ist deren „einfache" Nutzbarkeit}

Selbst wenn dies aktuell noch etwas nach Zukunftsmusik klingen mag und primär auf die Betreuung von PmD mit Typ-1Diabetes ausgerichtet ist, gibt es zunehmend Ideen für eine bessere Betreuung von PmD mit Typ-2-Diabetes mit digi- taler Unterstützung. Dabei geht es vorrangig um die Auswertung und Interpretation der im Alltag anfallenden Daten im Sinne von daraus resultierenden konkreten Handlungsempfehlungen, die die PmD selber umsetzen können. Bei dieser Patientengruppe geht es sehr wohl auch noch um die Analyse und Interpretation von Daten, die bei der BGSM anfallen, auch um die Insulintitration dadurch zu steuern. Solch eine Optimierung der Betreuung in den Wochen und Monaten zwischen den Arztvisiten in Form einer ,virtuellen Diabetesbetreuung “ (VDB) wird vielen PmD helfen und den Betreuungsaufwand für die Diabetesteams reduzieren [3].

Beiall diesen skizzierten Einsatzoptionen kommen Apps mit und ohne Internetanbindung ins Spiel, so können PmD Hilfe bei einer der wichtigsten Fehlerquellen im Bereich der Diabetestherapie bekommen, bei der Abschätzung des Kohlenhydratgehaltes in einer Mahlzeit. Es gibt verschiedene Ansätze, durch Fotografieren des Tellers mit verschiedenen Nahrungsmitteln dem PmD Aussagen zu den darin enthaltenen „Broteinheiten“ $\mathrm{zu}$ liefern. Dies kann sowohl lokal auf dem Smartphone erfolgen oder durch Anbindung ans Internet im Zugriff auf große Datenbanken und Rechenkapazitäten. Bei vergleichbaren Datenströmen werden die Daten von CGM-Systemen im Netz analysiert, und bei Auffälligkeiten wird der Nutzer alarmiert. Wenn dies gewünscht ist, können solche Alarme auch an Familienmitglieder oder Freunde gehen, die die PmD bei Bedarf nicht nur ansprechen können, sondern über die Ortung des Smartphones auch lokalisieren können, etwas was im Falle von schweren Hypoglykämien extrem hilfreich sein kann. Das Diabetesteam kann parallel da$\mathrm{zu}$ informiert werden und den $\mathrm{PmD}$ von sich aus kontaktieren.

\section{DiaDigital - Bewertung von Apps}

Eine recht heterogen zusammengesetzte Gruppe von Diabetologen, Patienten, Herstellern etc. hat seit Anfang 2015 ein Konzept zur Bewertung von Apps entwickelt $[4,5]$. Zur Vermeidung von rechtlichen Problemen erfolgt diese Bewer- tung im Sinne einer begleiteten Selbstauskunft, die im Endeffekt zur Vergabe eines Siegels der Diabetesverbände an die jeweilige App führt. DiaDigital nimmt keine Negativbewertungen vor, bei dem Bewertungsprozess kommt es aber zu einer Beratung der Hersteller/Vertreiber der Apps bei Fehlern und Defiziten.

Der Bewertungsprozess wird von dem Hersteller einer App initiiert, d. h. dieser bewirbt sich um das App-Siegel von DiaDigital. Er lädt sich das Selbstauskunftsformular runter und füllt es für die gängigen Betriebssysteme aus. Die DiaDigital-Gruppe prüft, ob die selbst erstellten Kodexkriterien erfüllt sind, und eine staatlich unterstützte Institution in Bochum (ZTG Zentrum für Telematik und Telemedizin $\mathrm{GmbH}$ ) führt eine technische Prüfung durch. Dann wird eine Bewertungsseite eingerichtet, und die Tester der DiaDigital-Gruppe werden eingeladen zur Bewertung der gegebenen App. Die Ergebnisse ihrer Testung tragen sie online in die Bewertungsmaske ein, dabei gibt es Auswahlfelder und freie Angaben. Es sollen immer mindestens 5 Betroffene und 5 Behandler die Testung einer App durchführen. Diese Vorgabe ist getrieben von dem Gedanken, dass nur Betroffene die sie betreffenden Apps wirklich beurteilen können. Insgesamt 170 Tester haben sich registriert. Im Rahmen einer Telefonkonferenz werden die Ergebnisse besprochen, und es wird entschieden, ob das Siegel vergeben werden kann, und ein gemeinsames Bewertungsfazit formuliert. Die bei DiaDigital gewährleistete Transparenz gibt allen Beteiligten und v. a. den Nutzern der Apps die Sicherheit, eine rechtlich und medizinisch geprüfte Lösung zu verwenden. $\mathrm{Da} z u$ Beginn die rechtlichen Bedenken hoch waren, hat die DDG (Deutsche Diabetes Gesellschaft e. V.) das Konzept von DiaDigital von Anwälten prüfen lassen.

Als Ergebnis des nun über einige Jahre bei einer Reihe von Apps durchgeführten Testablaufs haben 9 Apps ein Siegel erhalten, immerhin 8 Apps haben dies nicht bekommen! Dabei haben die abgelehnten Apps am meisten Arbeit gemacht, bei der Bewertung dieser Apps wurden auch ernsthafte Fehler und Risiken aufgedeckt. Diese Arbeit hat zu einer Reihe von relevanten Erkenntnissen ge- 
Hier steht eine Anzeige.

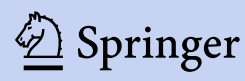


führt, dabei wurde ein Ziel von DiaDigital definitiv erreicht, d. h. die Kompetenz der Beteiligten zum Thema Apps wurde deutlich gesteigert, was auch dazu geführt hat, dass den Herstellern, aber auch den Anwendern geeignete Hilfestellungen bei der Verbesserung und Auswahl von Apps gegeben werden können. Selbstkritisch gilt es anzumerken: Die rasche Weiterentwicklung von Apps (s. oben) hat $\mathrm{zu}$ einer massiven Ausweitung der Komplexität von Apps geführt, diese sind meistens nicht mehr ein kleines Programm zur Dokumentation von Blutzuckerwerten, sondern vielfach stellt die App nur eine Schnittstelle zu einem hochkomplexen Medizinprodukt dar, so z.B. einem Boluskalkulator. Die Bewertung solcher Produkte ist nicht nur schwieriger, damit sind auch deutlich mehr Konsequenzen verbunden, d. h. Risiken für die Nutzer. Gleichzeitig steigt der Bewertungsaufwand wesentlich an, was für eine ehrenamtlich arbeitende Gruppe auf Dauer nicht leistbar ist. Diese Belastung hat auch dazu geführt, dass es zunehmend schwieriger wurde, Tester zu motivieren, die Apps zu bewerten.

In den Jahren wurde auch deutlich, einige Aspekte wurden bei der Entwicklung der Bewertungskriterien nicht ausreichend berücksichtigt und sich die Situation weiterentwickelt hat, wobei der eigentliche Kriterienkatalog recht konstant geblieben ist:

- Macht die App überhaupt Sinn?

- Wie ist deren Usability?

- Bewertung von Datenschutzproblemen

- Viele Entwicklungen sind mehr technologie- als anwendergetrieben.

Im Gesamtbild gilt es zu konstatieren, es fehlt die Einbindung in einen geeigneten rechtlichen und organisatorischen Rahmen, der die Arbeit von DiaDigital adäquat unterstützt und vorantreibt. Dies gilt es zu sehen von einem ausgesprochen positiven und großen Echo in den Medien zu dieser Aktivität, auch in der politischen Ebene.

Diabetologe 2021 · 17:275-282 https://doi.org/10.1007/s11428-020-00700-0

(c) Springer Medizin Verlag GmbH, ein Teil von Springer Nature 2020

\section{Heinemann · D. Drossel · M. Kaltheuner}

\section{DiaDigital, Apps und digitale Gesundheitsanwendungen. Resümee nach 5 Jahren und wie weiter?}

\section{Zusammenfassung}

Die COVID-Pandemie hat der Nutzung von digitalen Optionen bei der Betreuung von Patienten mit Diabetes einen massiven Schub verliehen; die bereits vorher verfügbare, gute Struktur dafür hat beachtlich rasch reagiert. Ob diese Entwicklung hinsichtlich der Nutzung von telemedizinischen Betreuungsoptionen, Online-Schulungen etc. als etabliert betrachtet werden kann, wird die Zukunft zeigen. Die Güte der Kommunikation der Patienten mit dem Diabetologen/dem Diabetesteam ist bei einer guten IT-

Ausrüstung auch mit digitalen Optionen gut. Die in diesem Beitrag diskutierten Ansätze und Projekte, z. B. Apps und deren Bewertung, die digitalen Gesundheitsanwendungen und die virtuelle Betreuung von Patienten zwischen den Arztvisiten, haben in dieser Hinsicht in den letzten Jahren den Boden für diese Entwicklung bereitet, unterstützt auch von den erheblichen Veränderungen in der Gesundheitspolitik zu diesen Themen. Die jetzt schon verfügbare und die absehbar zur Verfügung stehenden digitalen Optionen werden die gute und sichere Betreuung von Patienten weiterhin gewährleisten und voraussichtlich noch deutlich verbessern.

Schlüsselwörter

Digitalisierung · Virtuelle Diabetesbetreuung · Videokonferenzen - Telemedizin . Datenmanagement

\section{DiaDigital, apps and digital health applications. Résumé after 5 years and afterwards?}

\section{Abstract}

The COVID pandemic has given a massive boost to the use of digital options in the care of patients with diabetes. In Germany a good structure for patient care was already established before the pandemic and this structure was able to react remarkably quickly to the challenge. The future will show whether this development can be regarded as established with respect to the use of telemedicine, on-line training etc. The quality of communication between patients and the diabetologist/diabetes team can be sufficient with good IT equipment. The digital approaches and projects discussed in this article, e.g. apps and their evaluation, digital health applications and virtual care of patients between the visits, have prepared the ground for this development in recent years, supported also by the significant changes in health policy on these topics. The digital options that are already available and those that are likely to become available in the foreseeable future will continue to ensure good and safe patient care and are likely to significantly improve it.

\section{Keywords}

Digitalization - Virtual diabetes care . Video conferences $\cdot$ Telemedicine $\cdot$ Data management

\section{In welchem Ausmaß werden digitale Optionen in der Realität genutzt?}

Es gibt eher wenige belastbare Daten da$\mathrm{zu}$, in welchem Ausmaß PmD und Diabetologen/Diabetesteams digitale Optionen im Alltag in Deutschland wirklich nutzen, die aktuelle Pandemie hat hier mit Sicherheit zu massiven Verschiebungen geführt. Im Rahmen von jährlichen Umfragen über das Internet liefert der Digitalisierungs- und Technologie(D.U.T)Report Aussagen hierzu, wobei die Art der Datenerfassung einen gewissen Bias bedingt, $d$. h. es beantworten nur interessierte Ärzte, Beraterinnen und Patienten einen solchen Fragebogen, also die Interessierten und Motivierten [6]. Unter Berücksichtigung dieser Rahmenbedingungen ist in den letzten Jahren ein deutlicher Anstieg beim Interesse am Thema Digitalisierung und beim Einsatz von Diabetestechnologie (DT) in den Praxen zu konstatieren. 


\section{Nutzung von digitalen Optionen in Diabetesschwerpunktpraxen}

Es gibt keine öffentlich verfügbaren Statistiken dazu, welche Apps von welchen PmD wie intensiv genutzt werden. Interessant ist zu sehen, wenn die Motivation der PmD hoch ist, dann hat dies in dem hier interessierenden Sinne konkrete Auswirkungen, bei einer Auswertung in einer Diabetesschwerpunktpraxis (DSP) zeigte sich: $96 \%$ der Frauen, die zu einem oGTT (oraler Glukosetoleranztest) kommen, benutzen ein Smartphone. Während der Schwangerschaft benutzen diese Frauen z. T. mehrere Apps zur Begleitung ihrer Schwangerschaft. Der subjektive Nutzen bestimmt die Nutzung von digitalen Optionen. Dabei gilt es, die Motivation der Behandler nicht zu unterschätzen, bei der hier angesprochenen Patientengruppe ist diese vermutlich auch höher als bei anderen.

\section{Digitale Gesundheits- anwendungen}

Von der Definition her sind DiGAs (digitale Gesundheitsanwendungen) Medizinprodukte, die folgende Eigenschaften aufweisen: Sie fallen in die Risikoklassen I oder IIa, ihre Hauptfunktion beruht auf digitalen Technologien, sie sind keine digitalen Anwendungen, die lediglich dem Auslesen oder Steuern eines Gerätes dienen, der medizinische Zweck wird im Wesentlichen durch die digitale Hauptfunktion erreicht. In diesem Sinne unterstützt eine DiGA die Erkennung, Überwachung, Behandlung oder Linderung von Krankheiten oder die Erkennung, Behandlung, Linderung oder Kompensierung von Verletzungen oder Behinderungen, sie dient aber nicht der Primärprävention. Sie wird vom Patienten oder von Leistungserbringer und Patient gemeinsam genutzt, d.h. Anwendungen, die lediglich vom Arzt zur Behandlung der Patienten eingesetzt werden („Praxisausstattung"), sind keine DiGA. Die DiGA sollen "digitale Helfer" in der Hand der Patienten sein. DiGAs weisen mehr Nutzen durch mehr „Intelligenz“ auf, so durch den Einsatz von „,künstlicher Intelligenz".Die damit mögliche Datenanalyse kann zur Mustererkennung genutzt wer- den. Durch eine gemeinsame Kommunikationsplattform ist eine abgestimmte Therapieführung z.B. bei Frauen mit einem Gestationsdiabetes möglich.

Entsprechend den rechtlichen Vorgaben können sich nun Apps um die Aufnahme in das DiGA-Verzeichnis bewerben, um dann abrechenbar zu sein, d.h. der Arzt kann für deren Nutzung ein Rezept ausstellen, und der Hersteller bekommt eine Kostenerstattung. DiGAs erfahren eine vorläufige Aufnahme ohne Studie, der Hersteller hat dann 12 Monate Zeit, mit Studiendaten eine endgültige Aufnahme zu erreichen. Die DiGA (welche primär Apps sein werden) sollen eine CE-Markierung aufweisen, was als Nachweis zur Sicherheit und Funktionstauglichkeit gewertet wird. Wichtige Themen wie Datenschutz und Interoperabilität werden bei der Bewertung auch berücksichtigt. Es gilt, kritisch anzumerken in Anbetracht der Entwicklungsgeschwindigkeit von Apps: Wie schnell ist eine gegebene App, für die ein aufwendiger Zulassungsprozess durchlaufen wurde, nicht mehr aktuell, bzw. in welchem Ausmaß können Updates/Aktualisierungen einer App erfolgen, ohne dass diese eigentlich eine wesentlich geänderte/neue/andere App darstellt? Anders als bei Medikamenten und anderen Medizinprodukten ist die Entwicklungsgeschwindigkeit in dem Bereich „digitale tools" ausgesprochen hoch. Den ökonomischen Aufwand für die Erstellung einer App und deren $\mathrm{Zu}$ lassung als DiGA können sich nur wenige Hersteller leisten. Selbst wenn er beachtlich groß ist, ist der deutsche Markt alleine vermutlich nicht ausreichend, um die notwendigen Investitionen $\mathrm{zu}$ rechtfertigen. DiGA müssen so in das Therapiekonzept von Patienten eingebunden werden, dass der Patient und der Diabetologe davon einen Vorteil haben, nur dann wird der Diabetologe diese DiGA „verordnen“, und erst dann wird diese erfolgreich sein. Wie hoch die Kosten für DiGA sein werden, wird wohl erst nach den Verhandlungen zwischen den Kostenträgern und den Herstellern bekannt werden, wenn es denn wirkliche Transparenz hierbei geben wird, bei vielen anderen Medizinprodukten (z. B. CGM-Systemen) fehlt diese weitgehend.
Interoperabilitätsanforderungen an digitale Gesundheitsanwendungen

DiGAs müssen für die Aufnahme in das DiGA-Verzeichnis nachweisen, dass sie in Bezug auf 3 ausgewählte Fragestellungen interoperabel gestaltet sind:

- Sie erlauben es dem Versicherten, therapierelevante Auszüge der über die DiGA erhobenen Daten in menschenlesbarer und ausdruckbarer Form aus der DiGA auszugeben, sodass dieser diese zu eigenen Zwecken nutzen oder an einen Arzt weitergeben kann.

- Die DiGA erlaubt es dem Versicherten, die über die DiGA erhobenen Daten in einem maschinenlesbaren, interoperablen Format aus der DiGA auszulesen, sodass der Versicherte oder ein vom Versicherten berechtigter Dritter diese Daten über andere digitale Produkte weiterverarbeiten kann. Diese Schnittstelle soll perspektivisch auch an die gesetzlich vorgegebene elektronische Patientenakte (ePA) angebunden sein.

- Sofern die DiGA Daten aus vom Versicherten genutzten Medizingeräten oder vom Versicherten getragenen Sensoren zur Messung und Übertragung von Vitalwerten (Wearables) bezieht, kann sie diese Geräte auch über eine interoperable Schnittstelle ansprechen.

\section{Datenschutz}

Es gibt eine strenge Gesetzgebung zum Datenschutz in Deutschland und Europa. Dabei gilt es, die völlig nachvollziehbaren Ängste vor einer Verletzung der Privatsphäre zu sehen vor den Vorteilen, die eine gute Verfügbarkeit von Daten für die Patientenbetreuung und die wissenschaftliche Forschung bedeutet. Es gilt, zwischen diesen beiden Polen eine Haltung einzunehmen und zu lernen, Maßnahmen in deren Sinne zu ergreifen. Datenschutz darf nicht als Blockadegrund verwendet werden. 


\section{Änderungen und Empfehlungen}

Im Prinzip sind die Zukunftsaussichten für die Unterstützung bei der Diabetestherapie durch digitale Tools extrem gut, es stehen viel mehr Informationen zur Verfügung (nicht nur zu unmittelbar diabetesbezogenen Angaben) und Rechenpower! Für die PmD werden mehr und bessere Kommunikationsoptionen verfügbar werden, diese erhalten im Alltag konkrete Therapiehinweise, wenn ihre Therapie nicht sowieso automatisiert durch AID-Systeme geschieht. Der Diabetologe und sein Team bekommen Analysen aller Daten eines PmD, die sich daraus ergebenden Konsequenzen für Therapieanpassungen wurden im Hintergrund mit großen Datenbanken und Leitlinien abgeglichen. Die Kostenträger erhalten Informationen dazu, welche diagnostischen Maßnahmen und Therapien für den individuellen $\mathrm{PmD}$ am „effizientesten“ sind.

Die Einbindung solcher Optionen in die Behandlungsabläufe und -prozesse einer Praxis sind keine Trivialität, allerdings haben die Erfahrungen der letzten Monate gezeigt, wenn die Motivation - man kann auch sagen „der Druck“ hoch genug ist, dann geht dies erstaunlich schnell und gut. Die AGDT der DDG (Arbeitsgemeinschaft Diabetes \& Technologie der Deutschen Diabetes Gesellschaft) könnte viele Vollzeitstellen sinnvoll zur Unterstützung der , health care professionals" und natürlich der PmD und ihrer Verbände sinnvoll ausfüllen. Der Bedarf für eine fachlich kompetente Begleitung ist sehr groß, wie beispielhaft die obigen Ausführungen zu DiaDigital gezeigt haben.

Hier soll nicht unkritisch einer ,schönen neuen Welt" das Wort geredet werden, es gilt, die Vorbehalte und negativen Erfahrungen von Kollegen ernst zu nehmen, auch durch die Darstellung von Beispielen, bei denen die Digitalisierung der Praxis klar positive Ergebnisse gebracht hat. In den Praxen steckt der Teufel häufig im Detail, d.h. die verschiedenen Softwareprogramme blockieren sich gegenseitig, ein Update bei einem Programm führt zu Problemen bei anderen Programmen. Oder eine Firewall, die zur Absicherung vor Virenattacken un- abdingbar ist, blockiert den Transfer von Daten.

Viele Kollegen würden von konkreten Hilfestellungen durch Kollegen profitieren, d.h. Menschen, die keine eigenen ökonomischen Interessen verfolgen und sich mit den Abläufen in einer Praxis auskennen. Diese sollten im Sinne von Tutoren agieren. In vielen Fällen ist zunächst klarzumachen: „Digitalisierung“ einer Praxis ist nicht mit der Investition in neue Hard- und Software getan, es geht nicht um Maßnahmen, sondern um Änderungen in der generellen Herangehensweise und den Prozessen. Wenn es keine dazugehörige Honorierung durch die Kostenträger gibt, werden viele Kollegen solche Schritte nicht gehen wollen/ können! Keine Firma kann sich eine Investition ohne "return on investment" leisten, da stellen Arztpraxen keine Ausnahme dar. Wichtig ist in diesem Zusammenhang auch der Blick über den Tellerrand, d.h. welche Entwicklungen gibt es bei digitalen Tools für andere Indikationsbereiche und in anderen Ländern.

Das Anbieten von digitalen Tools alleine reicht nicht aus, es gilt, alle Beteiligten ausreichend in deren Nutzung zu schulen und Support bei Problemen anzubieten. Ohne Unterstützung bei der konkreten Nutzung z. B. einer DiGA werden viele $P m D$ diese im Alltag vermutlich nicht nutzen, d.h. der Aufwand für die Bereitstellung dieser Option war sinnlos. Können die Hotlines der Hersteller hier helfen? Die Erfahrungen von vielen PmwD mit Hotlines bei der Nutzung von Medizinprodukten sind wohl eher negativ. Bei den Verhandlungen zur Kostenerstattung muss für den Support auch ausreichend gesorgt werden! Der Support kann jedenfalls nicht auf die Praxen abgewälzt werden.

Bei vielen digitalen Tools fehlt ein eindeutiger Beleg für deren Sinnhaftigkeit durch geeignet angelegte klinische Studien. Die Kosten und der zeitliche Aufwand für die Schaffung von Evidenz ist allerdings beachtlich, auch vor dem Hintergrund einer sich rapide weiterentwickelnden Situation. Es gibt nur wenige „Klassenstudien" die in diesem Zusammenhang durchgeführt wurden [7]. Es wird notwendig sein, Belege für den Nutzen des Einsatzes von digitalen Tools aus deren Einsatz im Alltag abzuleiten. Ein Vorteil von solchen Tools ist, dass Versorgungsdaten quasi online zur Verfügung stehen. Es wird aber gelten, die verfügbaren Datenmengen entsprechend zu sichten und zu analysieren im Sinne von Big Data. Bei solchen Analysen sollten auch Nebenwirkungen gezielt untersucht werden, d.h. wo führt die Anwendung von solchen Tools nicht nur nicht zu dem gewünschten Ergebnis, sondern wo gibt es sogar negative Auswirkungen/wo ist die Investition in diese Tools nicht sinnvoll. Üblicherweise gibt es ein ausgeprägtes Underreporting von unerwünschten Wirkungen bei Medizinprodukten.

Es wird auch darum gehen zu analysieren, ob alle Patientengruppen in gleichem Ausmaß von dem Einsatz von digitalen Tools profitieren, ob es spezifische Barrieren für deren Einsatz bei speziellen Patienten gibt. Die Anwenderfreundlichkeit, d.h. die Barrierefreiheit, kann für die erfolgreiche Nutzung solcher Tools ein entscheidendes Kriterium sein. Wenn „Randgruppen“ existieren, dann gilt es, diese zu erkennen und zu schauen, wie diese gezielt angesprochen werden können.

\section{》) Kritische Faktoren für die Nutzung von digitalen Tools sind Zeit und Aufwand für deren Nutzung}

Es ist davon auszugehen, dass sich der Umgang mit digitalen Tools in naher Zukunft ändern wird, ein „Herumtippen" auf dem Smartphone wird vermutlich durch eine Sprachsteuerung abgelöst werden. Dafür wird eine gewisse Standardisierung der Nutzeroberflächen notwendig sein.

Es wirkt so, als ob die Hersteller von Medizinprodukten eher zurückhaltend sind, neue Versionen ihrer Apps/ Software auf den Markt zu bringen, dies gilt nicht für die eigentlichen technischen Produkte, da kommen Generationswechsel schneller. Fehlt hier der Druck von den Nutzern zu rascheren Anpassungen? Dabei sollten Diabetologen und Mitglieder des Diabetesteams früher und stärker in die Entwicklungsprozesse 
Hier steht eine Anzeige.

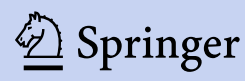


eingebunden werden, v. a. sollten diese die Besonderheiten des deutschen Gesundheitssystems stärker berücksichtigen. Ein kritischer Faktor für die Nutzung von digitalen Tools sind die Zeit und der Aufwand für deren Nutzung. Wenn die Nutzer (hier liegt der Fokus auf den Diabetologen/Diabetesteams) keinen Vorteil darin sehen, erfolgt die Nutzung nicht.

Im Bereich der Diabetologie (in anderen Fachgebieten vermutlich auch) stellt die An- und Einbindung von digitalen Tools an die Praxisverwaltungssoftware eine kritische Hürde dar. Ob die Einführung von ePA und elektronische Diabetes-Akte (eDA) hier zu positiven Veränderungen führen wird, bleibt abzuwarten. Wenn in Zukunft neue Projekte, Initiativen etc. zur Verbesserung der Behandlung von PmD vorgestellt werden, dann sollte dabei der Aspekt einer ausreichenden Kommunikation und Einbindung aller relevanten Gruppen mehr Raum bekommen, insbesondere das Mitnehmen derjenigen, die die Dinge in den Praxen konkret machen sollen. Eine Diskussion mit vielen Beteiligten kostet Zeit und Kraft, führt aber auch dazu, dass sie diese Entwicklungen mittragen. Ein „Überstülpen“ von digitalen Tools wird in vielen Fällen zum Gegenteil des Beabsichtigten führen.

\section{Fazit für die Praxis}

- Die aktuelle Pandemie hat der Nutzung von digitalen Optionen bei der Betreuung von Patienten mit Diabetes einen wesentlichen Schub gegeben.

- Sowohl die Patienten wie auch die Diabetesteams nutzen zunehmend Apps und andere Softwareoptionen für das Datenmanagement.

- Es gilt, den Praxen eine geeignete Unterstützung bei der Bewertung der unterschiedlichen Optionen zu geben. Hier sind sowohl die Fachgesellschaft wie auch die entsprechenden Arbeitsgemeinschaften und Kommissionen gefordert.

- In Zukunft werden für Diabetestherapie wesentlich mehr digitale Tools zur Verfügung stehen. Durch geeignete Analyse der Vielzahl an verfügbaren
Informationen erhalten die Patienten konkrete Therapiehinweise, wenn deren Therapie nicht sowieso automatisiert erfolgt.

\section{Korrespondenzadresse}

Prof. Dr. Lutz Heinemann
Science Consulting in
Diabetes GmbH
Geulenstr. 50, 41462 Neuss,
Deutschland
Lutz.Heinemann@profil.com

\section{Einhaltung ethischer Richtlinien}

Interessenkonflikt. L. Heinemann, D. Drossel und M. Kaltheuner geben an, dass kein Interessenkonflikt besteht.

Für diesen Beitrag wurden von den Autoren keine Studien an Menschen oder Tieren durchgeführt. Für die aufgeführten Studien gelten die jeweils dort angegebenen ethischen Richtlinien.

\section{Literatur}

1. Ahn DT, Stahl R (2019) Is there an app for that? The pros and cons of diabetes smartphone apps and how to integrate them into clinical practice. Diabetes Spectr 32(3):231-236

2. Fleming GA, Petrie JR, Bergenstal RM, Holl RW, Peters AL, Heinemann L (2020) Diabetes digital app technology: benefits, challenges, and recommendations. A consensus report by the European association for the study of diabetes (EASD) and the American diabetes association (ADA) diabetes technology working group. Diabetes Care 43(1):250-260

3. Phillip M, Bergenstal RM, Close KL, Danne T, Garg S, Heinemann L et al (2020) The digital/virtual diabetes clinic: the future is now - recommendations from an international panel on diabetes digital technologies introduction. Diabetes Technol Ther. https://doi.org/10.1089/dia.2020.0375

4. Kaltheuner M, Drossel D, Heinemann L (2019) DiaDigital Apps: evaluation of smartphone apps using a quality rating methodology for use by patients and diabetologists in Germany. J Diabetes Sci Technol 13(4):756-762

5. Kaltheuner MD, Droßel D, Heinemann L (2016) DiaDigital - Unterstützung von Patienten und Diabetologen bei der Nutzung von Apps. Diabetologe. https://doi.org/10.1007/s11428-016-0162-x

6. Kulzer BH, Heinemann L, Hochstadt S, Keuthage W, Kröger J, Lueg A, Mühlen HJ, Schütte L, Ehrmann D, Hermanns N, Roos T (2019) Wie digital ist die Diabetologie in Deutschland? Ergebnisse des Digitalisierungs-und Technologiereports Diabetes 2019. Diabetes Stoffwechs Herz 28:189-196

7. Kulzer B, Daenschel W, Daenschel I, Schramm W, Messinger D, Weissmann J, Vesper I, Parkin C, Heinemann L (2018) Integrated personalized diabetes management improves glycemic contro in patients with insulin-treated type 2 diabetes: Results of the PDM-ProValue study program.
Diabetes Res Clin Pract. https://doi.org/10.1016/j. diabres.2018.09.002 